\title{
Bilarge Mixing of Leptons Using Abelian Flavor Symmetries
}

\author{
T. Ohlsson ${ }^{\text {a* }}$ and G.Seidl ${ }^{\text {a }}$
}

anstitut für Theoretische Physik, Physik-Department, Technische Universität München, James Franck-Straße, 85748 Garching bei München, Germany

In order to understand the leptonic masses and mixing parameters in terms of approximately conserved underlying flavor (or horizontal) symmetries, we extend the standard model (SM) by a set of fundamental heavy fermions and SM singlet scalars and assign the fermions as well as the scalars a number of horizontal $\mathbb{Z}_{n}$ and $U(1)$ charges $[1,2]$. Then, the lepton masses are generated by non-renormalizable terms via the Froggatt-Nielsen mechanism [3]. Among the flavor symmetries are $\mathbb{Z}_{2}$ permutation symmetries which establish exact relations between the Yukawa couplings and the couplings in the multiscalar potential (and yield in total a non-Abelian symmetry group). We assume that the vacuum expectation values of the SM singlet scalars are all of the same order of magnitude and hence give rise to a common flavor symmetry breaking parameter $\epsilon \simeq 10^{-1}$. For a range of parameters a vacuum alignment mechanism generates charged lepton and neutrino mass matrices $M_{\ell}$ and $M_{\nu}$ of the forms

$$
\begin{aligned}
& M_{\ell}=m_{\tau}\left(\begin{array}{ccc}
\epsilon^{3} & \epsilon^{2} & \epsilon^{4} \\
\epsilon^{3} & \epsilon & \epsilon^{2} \\
\epsilon^{3} & \epsilon^{2} & 1
\end{array}\right), \\
& M_{\nu}=m_{\nu}\left(\begin{array}{ccc}
\epsilon^{2} & 1 & -1 \\
1 & \epsilon^{4} & \epsilon^{4} \\
-1 & \epsilon^{4} & \epsilon^{4}
\end{array}\right),
\end{aligned}
$$

where $m_{\tau}$ is the tau mass and $m_{\nu}$ is the absolute neutrino mass scale. The important and novel feature of the model is that the flavor symmetries enforce in Eq. (2) the entries " -1 " and "1" to be practically exactly degenerate, which naturally gives a close to maximal atmospheric mixing

*E-mail: tohlsson@ph.tum.de

†E-mai: gseidl@ph.tum.de angle

$\theta_{23}=\pi / 4+\mathcal{O}\left(\epsilon^{2}\right)$.

Assuming no CP-violation, we obtain for the solar mixing angle $\theta_{12}$ and the reactor mixing angle $\theta_{13}$ the relation

$\theta_{12}=\pi / 4-\theta_{13}+\mathcal{O}\left(\epsilon^{2}\right)$,

where $\theta_{13} \simeq \epsilon / \sqrt{2}+\mathcal{O}\left(\epsilon^{3}\right)$. The model gives the strictly hierarchical charged lepton mass ratios $m_{e} / m_{\mu} \simeq \epsilon^{2}$ and $m_{\mu} / m_{\tau} \simeq \epsilon$, and an inverted neutrino mass hierarchy with mass squared differences $\Delta m_{\odot}^{2} \simeq 2 \sqrt{2} \epsilon^{2} m_{\nu}^{2}$ and $\Delta m_{\text {atm }}^{2} \simeq 2 m_{\nu}^{2}$. Using $m_{\nu} \simeq 0.04 \mathrm{eV}$ and $\epsilon \simeq 10^{-1}$ we obtain the mass squared differences $\Delta m_{\odot}^{2} \simeq 10^{-5} \mathrm{eV}^{2}$ and $\Delta m_{\text {atm }}^{2} \simeq 10^{-3} \mathrm{eV}^{2}$ of the presently preferred Mikheyev-Smirnov-Wolfenstein (MSW) large mixing angle (LMA) solution of the solar neutrino problem [4]. In total, the mixing angles are

$\theta_{12} \simeq 41^{\circ}, \quad \theta_{13} \simeq 4^{\circ}$, and $\theta_{23} \simeq 44^{\circ}$,

i.e., the solar mixing angle is large but not necessarily close to maximal, the reactor mixing angle is small and consistent with the CHOOZ upper bound, and the atmospheric mixing angle is close to maximal, as required by the MSW LMA solution [4].

\section{REFERENCES}

1. T. Ohlsson and G. Seidl, Phys. Lett. B 537 (2002) 95.

2. T. Ohlsson and G. Seidl, hep-ph/0206087.

3. C. D. Froggatt and H.B. Nielsen, Nucl. Phys. B 147 (1979) 277.

4. J. N. Bahcall, M. C. Gonzalez-Garcia, and C. Peña-Garay, hep-ph/0204314. 